\title{
Erosion versus recurrence: Is there a compromise using biologics for ventral rectopexy?
}

\author{
A. R. L. Stevenson
}

Received: 20 February 2015/Accepted: 21 February 2015/Published online: 3 March 2015

(C) Springer-Verlag Italia Srl 2015

Of the many surgical options available for the treatment of rectal prolapse, ventral rectopexy (VR) has recently gained in popularity in various parts of the world. There have been a large number of studies reported since D'Hoore and Penninckx published their results for rectal prolapse using a minimally invasive technique in 2004. This has been successfully used in various centres, not only for the treatment of external rectal prolapse, but also for internal prolapse, rectocele and even solitary rectal ulcer syndrome $[1,2]$. Whilst the concept of surgical repair of internal prolapse remains controversial, there is no doubting that the results reported seem to have been effective in a large number of patients with minimal morbidity.

Deucher first used the term "ventral rectopexy" with a dissection solely in the anterior plane in 1960 [3]. Initial descriptions by Orr-Loygue involved both anterior and posterior mobilisation and mesh fixation. Others had also performed this technique in the 1980s, but it was really with the introduction of laparoscopic surgery and the excellent results reported by D'Hoore and others that the technique has gained popularity.

Ventral rectopexy has been slow to be adopted in several parts of the world, particularly the USA. Apart from the technical aspects of suturing within a confined space, the main concern has been the use of synthetic mesh within the pelvis, risking erosion, infection and chronic pain. This concern has arisen largely in the gynaecological literature, leading to the FDA issuing a warning about the use of synthetic mesh in the pelvis for pelvic organ prolapse repair in 2011. Indeed, there are now tens of thousands of

\footnotetext{
A. R. L. Stevenson ( $₫)$

Department Colorectal Surgery, Royal Brisbane Hospital,

University of Queensland, Brisbane, Australia

e-mail: arl.stevenson@me.com
}

multi-district litigation lawsuits concerning synthetic mesh used for pelvic organ prolapse submitted to the US Federal Courts. Over the last 3 years, there has been an exponential growth, particularly in law suits, as well as settlements, amounting to over US\$1 billion.

This has led to a number of medical device companies withdrawing their products from the market. Not surprisingly, it has also contributed to a sense of caution by many colorectal surgeons when considering the utility of VR for treatment of their patients with prolapse. Furthermore, concerns persist as to how the deep placement of a synthetic mesh may affect subsequent treatment for future rectal cancers [4].

This concern about the risks of synthetic mesh has led some groups to using biologic grafts to effect the repair. In this issue of Techniques in Coloproctology, Franceschilli and Sileri have been using one type of biologic mesh and report a critical appraisal of their first one hundred patients for the treatment of obstructed defaecation and/or faecal incontinence in patients with internal rectal prolapse.

Franceschilli et al. used Permacol, which is a porcinederived dermal collagen, which has been cross-linked to supposedly increase the length of time the graft remains in situ. Fixation to the distal rectum was done using permanent sutures, absorbable sutures to the vaginal vault and tacks to the sacral promontory. The peritoneum was then closed with a barbed absorbable suture (V-lok).

Whilst this is a relatively short follow-up with the mean of 20 months (range 6-54 months), this study adds support to the use of biologic material as a viable alternative to synthetic mesh for the treatment of pelvic organ prolapse. The functional outcomes reported by this group are certainly acceptable with the improvement in incontinence of $86 \%$. Similarly, symptoms of constipation and obstructed defaecation also improved in $92 \%$ of patients. There was 
no worsening of continence status, constipation nor sexual function observed. These results are certainly comparable with previously reported outcomes using synthetic mesh. However, 14 patients $(14 \%)$ experienced persistent symptoms or recurrence of prolapse.

The rate of recurrence may seem slightly higher than previously reported series of around $4 \%$; there may also be technical aspects to explain these results rather than simply the type of mesh or graft. Many surgeons will also use sutures to the mid-rectum, as well as the distal attachment to the rectum and levator. It was also noted that the majority of the persistent or recurrent prolapse was early on in the learning curve, within the first 20 patients. There were no reports of mesh-related complications, but two small bowel obstructions. These were said to be related to attachment to the tack in the sacral promontory where the peritoneum had not been completely closed. However, it is interesting to note the reports appearing in the literature relating to small bowel obstruction due to V-lok suture, particularly in cases of ventral rectopexy [5].

Whilst previous studies in the gynaecologic literature had shown inferior functional outcome results with biologic graft compared with synthetic mesh, there has been wide variation in techniques and the type of graft utilised. Additionally, there have been a number of advances made in the development of biologic grafts over the past two decades. This makes it quite difficult for the surgeon in terms of choice of prosthesis, particularly with the lack of large randomised trials comparing modern grafts and synthetic mesh. Indeed, it may not be simply the type of mesh employed, but also the method of dissection and attachment, particularly with the use of permanent sutures. There is recommendation that permanent, braided sutures be avoided for the fixation of mesh to the rectum or vagina [6]. Alternatives such as absorbable sutures or even biologic glue or tissue glue may also be viable. However, it is difficult to know whether this may influence the functional results or recurrence of prolapse.

There have also been reports of erosion using Permacol mesh for ventral rectopexy. It is difficult to know whether this is due to the type of cross-linked biologic graft or whether there were other technical issues including the use of permanent, braided sutures.
Treatment of patients with symptoms of incontinence or obstructed defaecation due to internal prolapse and rectocele remains controversial. However, it would appear that many of these patients can have improvement in their symptoms with a technique that is minimally invasive and with good short-term outcomes and minimal morbidity. Limited data in the use of biologic grafts for VR would suggest similar effectiveness in the short term, compared with synthetic mesh [7-9]. If longer-term studies also demonstrate that the use of biologic graft provides equivalent functional results, there may be the additional benefit of lower risk of erosion or infection in the treatment of pelvic organ prolapse.

Conflict of interest Prof. Stevenson has been a proctor for Applied Medical, Cook Medical, Johnson \& Johnson.

\section{References}

1. Wong M, Meurette G, Abet E, Podevin J, Lehur PA (2011) Safety and efficacy of laparoscopic ventral mesh rectopexy for complex rectocele. Colorectal Dis 13:1019-1023

2. Badrek-Amoudi AH, Roe T, Mabey K, Carter H, Mills A, Dixon AR (2013) Laparoscopic ventral mesh rectopexy in the management of solitary rectal ulcer syndrome: a cause for optimism? Colorectal Dis 15:575-581

3. Deucher F (1960) Ventral rectopexy in the treatment of rectal prolapse. Helv Chir Acta 27:240-246

4. Antonowicz SS, Al-Whouhayb M, Middleton S (2013) Total mesorectal excision for cancer following ventral mesh rectopexy. Ann R Coll Surg Engl 95:e95-e96

5. Salminen HJ, Tan WS, Jayne DG (2014) Three cases of small bowel obstruction after laparoscopic ventral rectopexy using the V-Loc $\left({ }^{\circledR}\right)$ suture. Tech Coloproctol 18:601-602

6. Mercer-Jones MA, D'Hoore A, Dixon AR et al (2014) Consensus on ventral rectopexy: report of a panel of experts. Colorectal Dis 16:82-88

7. Smart NJ, Pathak S, Boorman P, Daniels IR (2013) Synthetic or biological mesh use in laparoscopic ventral mesh rectopexy a systematic review. Colorectal Dis 15:650-654

8. Ogilvie JW Jr, Stevenson AR, Powar M (2014) Case-matched series of a non-cross-linked biologic versus non-absorbable mesh in laparoscopic ventral rectopexy. Int $\mathrm{J}$ Colorectal Dis 29:1477-1483

9. Wahed S, Ahmad M, Mohiuddin K, Katory M, Mercer-Jones M (2012) Short-term results for laparoscopic ventral rectopexy using biological mesh for pelvic organ prolapse. Colorectal Dis 14:1242-1247 